\title{
Describing heavy-ion fusion with quantum coupled-channels wave-packet dynamics
}

\author{
Terence Vockerodt and Alexis Diaz-Torres (1) \\ Department of Physics, University of Surrey, Guildford GU2 7XH, United Kingdom
}

(Received 23 July 2019; published 6 September 2019)

\begin{abstract}
We investigate the fusion of ${ }^{16} \mathrm{O}$ and ${ }^{154} \mathrm{Sm}$ with excited states at Coulomb energies using a theoretical dynamical model. The two-body Schrödinger equation is solved using the time-dependent wave-packet coupledchannels method. The wave function of the collective motion and excitations are visualized in both position and momentum space, providing a detailed mechanism of the reaction. We benchmark our calculations of the energy-resolved fusion transmission coefficients with those from the time-independent coupled-channels method. The present results are in good agreement with the time-independent results for a wide range of energies and angular momenta, demonstrating both the reliability of the quantum wave-packet dynamical approach for fusion and its usefulness for providing additional insights into fusion dynamics.
\end{abstract}

DOI: 10.1103/PhysRevC.100.034606

\section{INTRODUCTION}

Time-dependent simulations can give unique physical insights into many systems of interest such as nuclear reactions. The ability to monitor the exact chain of events the system undergoes makes sensible conclusions of the efficacy of theoretical models extremely tractable [1]. The price paid in computational time (relative to time-independent methods) to perform these time-dependent calculations can yield some useful information, such as the deduction of intermediate states and competition of mechanisms. Whereas it is possible to return to the time-dependent picture after the energy eigenstates are generated from time-independent methods, the time-dependent results from this method can only be found for time-independent Hamiltonians for specific initial states, which limits the range of phenomena that they can model. For example, if one wishes to model a process with a spatially localized initial state, then this may require that an impractical number of energy eigenstates must be found to construct the superposition, whereas in time-dependent methods any initial state can be propagated in time.

Crucially, there has been a large effort in modeling dissipative processes with dynamical approaches [2]. These involve either a directly evaluated dissipation interaction (such as the Caldeira-Leggett model and its ilk [3]) or the averaging of the effects of stochastic interactions with finite baths (as is used in the stochastic surrogate Hamiltonian approach $[2,4]$ ). The time propagation techniques that use these dissipative Hamiltonians and/or master equations have also been well studied (see Ref. [2] and references therein). The key strength of these polynomial propagators is the fidelity of the propagation relative to the exact solution, which for example allows one to distinguish between the loss of norm due to the computational error and the loss of norm due to dissipation and/or absorption with a very high degree of accuracy. This is due to numerical errors not accumulating, even with a high number of polynomials [2].

An example of an effective dynamical approach for scattering reactions is the time-dependent wave-packet (TDWP) approach, which has been successfully implemented to describe chemical reactions [5-8], as well as in nuclear physics [9-12]. Typically in these approaches, a spatially localized wave packet is chosen as the initial state. Then, after the time propagation, relevant energy-resolved observables such as cross sections are obtained using energy projection methods on the initial and final wave packets. This combination allows for modestly sized grids in position to be used for the entire propagation over time while minimizing the number of propagations needed to generate results (since propagating a spatially localized wave packet achieves the same results as a parallel propagation of multiple energy states, provided one can accurately find the energy-resolved final states). The use of coordinate grids allows for more practical simulations of effects such as breakup and incomplete fusion for three-body systems, which require high partial wave expansions that exceed the cutoff for some time-independent models [10].

In this paper, we demonstrate the effectiveness of the timedependent coupled-channels wave-packet (TDCCWP) quantum dynamical model as applied to nuclear scattering between an inert ${ }^{16} \mathrm{O}$ projectile and a ${ }^{154} \mathrm{Sm}$ target with a rotational band, in the body-fixed center-of-mass frame. We benchmark our calculations of the fusion transmission coefficients against those from the time-independent coupled-channels approach [13].

The paper layout is as follows: Section II introduces the propagator and energy projection methods used, as well as the Hamiltonian that we consider. Section III gives the inputs used for the model, discusses the quality of the propagated wave functions, and compares the energy-resolved transmission coefficients between the time-dependent and time-independent models. Finally, Sec. IV concludes with a summary of the results.

\section{MODEL AND METHODS}

\section{A. TDCCWP method and the Fourier grid}

There are three steps in the TDWP method $[1,11,12]$ : 
(i) The initial wave function $\psi\left(t=t_{0}\right)$ is generated.

(ii) The initial wave function is propagated to the desired final time $t_{f}$ using the time evolution operator.

(iii) The final state $\psi\left(t_{f}\right)$ is extracted and used to calculate observables such as spectra, transmission coefficients, and cross sections.

The wave function and the spatial parts of the Hamiltonian $\hat{H}$ are represented in a Fourier grid [1] using only the internuclear distance $r$. The propagation is achieved using a Chebyshev polynomial expansion [12,14]. After a long time propagation, where the wave packet is in the asymptotic region far from the reaction center, the results we generate using the time-dependent Schrödinger equation should be equivalent to those of the time-independent Schrödinger equation (TISE).

\section{B. Initial wave function and parameters}

The internuclear distance wave function at $t=0$ is expressed as a Gaussian wave packet, with an initial boost towards $r=0$ :

$$
\psi_{0}(r)=\frac{1}{\pi^{1 / 4} \sqrt{\sigma}} \exp \left(-\frac{\left(r-R_{0}\right)^{2}}{2 \sigma^{2}}\right) \exp \left(-\mathrm{i} k_{0} r\right)
$$

where $\sigma$ is the spatial width of the wave packet, $R_{0}$ is the starting position of the centroid, and $k_{0}$ is the average wave number for the incident wave packet, which depends on the incident energy $E_{0}$ and $\sigma$ and is found by solving $E_{0}=$ $\left\langle\psi_{0}|\hat{H}| \psi_{0}\right\rangle$.

\section{The coupled-channels Hamiltonian}

For a scattering reaction between two nuclei in the bodyfixed center-of-mass frame with absorption near the reaction center, the coupled-channels equation is given by [13]

$$
\begin{aligned}
\mathrm{i} \hbar \frac{\partial \psi_{n}(r)}{\partial t}= & \left(\frac{-\hbar^{2}}{2 \mu} \frac{d^{2}}{d r^{2}}+\frac{J(J+1) \hbar^{2}}{2 \mu r^{2}}+V(r)+\epsilon_{n}\right) \psi_{n}(r) \\
& +\sum_{m} V_{n m}(r) \psi_{m}(r),
\end{aligned}
$$

where $r$ is the distance between the two nuclei, $\mu$ is the reduced mass, $J$ is the total angular momentum, and $V(r)=$ $U(r)+\mathrm{i} W(r)$ is the sum of the real nuclear potential $U(r)$ and a short range absorption potential $W(r)$. Propagating the wave packet in the body-fixed frame is more efficient than propagating in the space-fixed (laboratory) frame, since it reduces the dimensionality of the wave packet. Equation (2) employs the iso-centrifugal approximation, which neglects the interaction between the orbital angular momentum and the intrinsic spin of the nuclei. The absorption provided by the imaginary potential $W(r)$ is to include the irreversibility of the fusion process, by removing flux from waves inside the fusion pocket. $U(r)$ is the sum of the Woods-Saxon and Coulomb potentials,

$$
U(r)=-\frac{V_{W S}}{1+\exp \left(\left(r-R_{W S}\right) / a\right)}+\frac{Z_{P} Z_{T} e^{2}}{r},
$$

where $R_{W S}=r_{W S}\left(A_{P}^{1 / 3}+A_{T}^{1 / 3}\right), a$ is the range of the potential in femtometers, and $Z_{P}$ and $Z_{T}$ are the charges of the

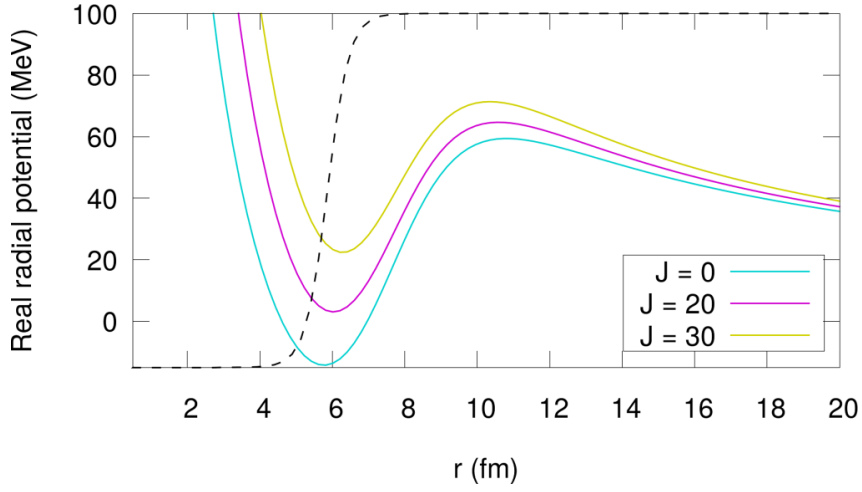

FIG. 1. Radial potentials for varying $J$. The cyan (lowest, gray), magenta (middle, dark grey), and yellow (highest, light gray) lines correspond to the potentials for $J=0,20$, and 30, respectively. The location of the absorption potential for $J=0$ is shown as a black dashed line (absorption potential energy values not to scale).

projectile and target, respectively. We show the real potentials for varying $J$ in Fig. 1.

The absorption potential takes a Woods-Saxon form:

$$
W(r)=-\frac{W_{0}}{1+\exp \left(\left(r-R_{\text {pock }}\right) / a_{W}\right)},
$$

where $R_{\text {pock }}$ is the location of the pocket of the nuclear potential, or the shortest distance where the nuclear potential in Eq. (3) is minimized. This absorption potential leads to loss of flux of the collective motion, and the potential physically represents degrees of freedom not accounted for in the Hamiltonian, such as complex non-collective excitations in the compound nucleus.

\section{Coupling matrix elements}

In this work, the ${ }^{16} \mathrm{O}$ projectile is modeled as inert and the ${ }^{154} \mathrm{Sm}$ target has a rotational band [15]. We include only the quadrupole and hexadecapole modes, and use the rigid rotor model to describe the energy levels. The rigid rotor energies are given by [16]

$$
\epsilon_{I}=\frac{\hbar^{2} I(I+1)}{2 \mathcal{I}},
$$

where $I$ is the rotational state spin and $\mathcal{I}$ is the moment of inertia. The rotations are modeled as a deformation in the effective radius of the target, which modifies the WoodsSaxon potential [13],

$$
V_{N}(r, \hat{O})=-\frac{V_{W S}}{1+\exp \left(\left(r-R_{W S}-\hat{O}\right) / a_{W S}\right)},
$$

where $\hat{O}$ is the deformation on the unperturbed radius $R_{W S}$. The quadrupole and hexadecapole terms are contained in $\hat{O}$. The nuclear coupling matrix elements are evaluated as

$$
V_{N, n m}(r)=\sum_{\alpha}\langle n \mid \alpha\rangle\langle\alpha \mid m\rangle V_{N}\left(r, \lambda_{\alpha}\right)-V_{N}(r, 0) \delta_{m}^{n},
$$

where $\hat{O}|\alpha\rangle=\lambda_{\alpha}|\alpha\rangle$ and the last term prevents double counting of the Woods-Saxon potential when $n=m$. In the rotational basis, the matrix elements of the operator $\hat{O}$ are 
TABLE I. Model parameters used exclusive to the present methodology (Fourier grid, wave packet, imaginary potential, and window operator).

\begin{tabular}{lcc}
\hline \hline Variable & Value & Description \\
\hline NGP & 2048 & Number of grid points in the Fourier grid \\
$r_{\min }(\mathrm{fm})$ & 0.5 & Minimum value of $r$ in the Fourier grid \\
$r_{\max }(\mathrm{fm})$ & 500 & Maximum $r$ in Fourier grid \\
$\Delta t(\mathrm{~s})$ & $10^{-22}$ & Propagator time step \\
$R_{\text {ini }}(\mathrm{fm})$ & 200 & Initial position of wave packet \\
$\sigma(\mathrm{fm})$ & 10 & Width parameter of initial wave packet \\
$W_{0}(\mathrm{MeV})$ & 50 & Imaginary potential well height \\
$a_{W}(\mathrm{fm})$ & 0.3 & Imaginary potential well range \\
$n$ & 2 & Window operator overlap parameter \\
$\epsilon(\mathrm{MeV})$ & 0.5 & Window operator resolution parameter \\
\hline \hline
\end{tabular}

given by

$$
\langle n|\hat{O}| m\rangle=R_{T}\left(\beta_{2} \mathcal{F}(2, n, m)+\beta_{4} \mathcal{F}(4, n, m)\right),
$$

where $R_{T}=r_{\text {coup }} A_{T}^{1 / 3}, r_{\text {coup }}$ is the coupling radius, $\beta_{2}$ and $\beta_{4}$ are the deformation parameters for the quadrupole and hexadecapole modes, respectively, and

$$
\mathcal{F}(I, n, m)=\sqrt{\frac{(2 I+1)(2 n+1)(2 m+1)}{4 \pi}}\left(\begin{array}{ccc}
n & I & m \\
0 & 0 & 0
\end{array}\right)^{2} .
$$

We also include the deformed target Coulomb interaction, up to second order in the expansion of $\beta_{2}$ and first order in $\beta_{4}$. This term is given by

$$
\begin{aligned}
V_{C, n m}(r)= & \frac{3 Z_{P} Z_{T}}{5} \frac{R_{T}^{2}}{r^{3}}\left(\beta_{2}+\frac{2}{7} \sqrt{\frac{5}{\pi}} \beta_{2}^{2}\right) \mathcal{F}(2, n, m) \\
& +\frac{3 Z_{P} Z_{T}}{9} \frac{R_{T}^{4}}{r^{5}}\left(\beta_{4}+\frac{9}{7 \sqrt{\pi}} \beta_{2}^{2}\right) \mathcal{F}(4, n, m) .
\end{aligned}
$$

The total interaction $V_{n m}$ in Eq. (2) is given by the sum of the nuclear and Coulomb components. In the rotational band, we include the $0^{+}, 2^{+}$, and $4^{+}$states in our calculations.

TABLE II. Model parameters pertaining to the nuclei of the present Hamiltonian. Wood-Saxons and rotational parameters obtained from Ref. [15].

\begin{tabular}{lcc}
\hline \hline Variable & Value & Description \\
\hline$Z_{P}$ & 8 & Projectile proton number \\
$A_{P}$ & 16 & Projectile nucleon number \\
$Z_{T}$ & 62 & Target proton number \\
$A_{T}$ & 154 & Target nucleon number \\
$V_{W S 0}(\mathrm{MeV})$ & 165 & Wood-Saxons well depth \\
$r_{W S}(\mathrm{fm})$ & 0.95 & Wood-Saxons well center \\
$a(\mathrm{fm})$ & 1.05 & Wood-Saxons well range \\
$\epsilon_{2}(\mathrm{keV})$ & 82 & Second excited state energy $\left(2^{+}\right)$ \\
$\beta_{2}$ & 0.322 & Quadrupole deformation parameter \\
$\beta_{4}$ & 0.027 & Hexadecapole deformation parameter \\
$r_{\text {coup }}(\mathrm{fm})$ & 1.06 & Rotational coupling radius \\
\hline \hline
\end{tabular}
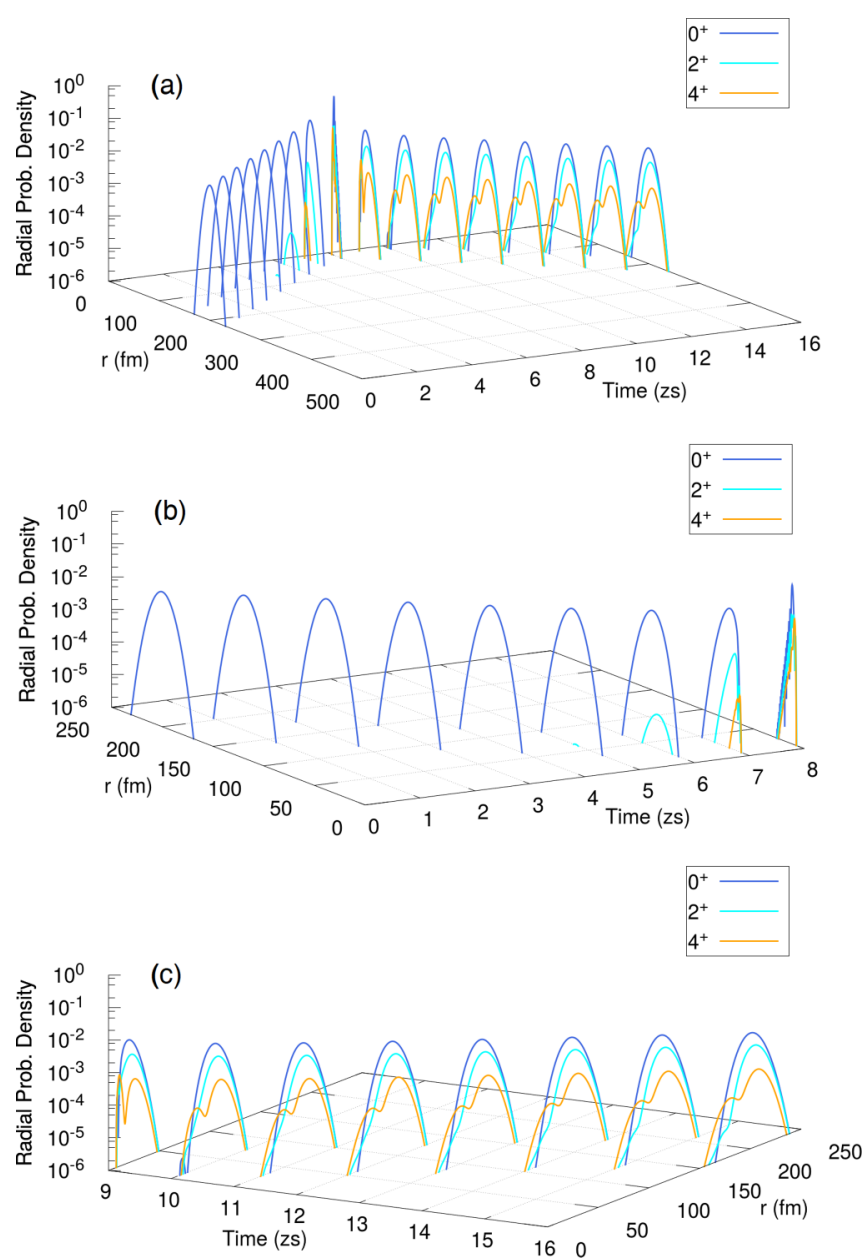

FIG. 2. Evolution of the radial probability density (a) over the whole time period, (b) from 0 to $8 \mathrm{zs}$ (incoming), and (c) from 9 to $16 \mathrm{zs}$ (outgoing), for $J=0$ and $E_{0}=60 \mathrm{MeV}$ for the ${ }^{16} \mathrm{O}+{ }^{154} \mathrm{Sm}$ collision. The blue (dark grey), cyan (light grey), and orange (grey) distributions are the $0^{+}, 2^{+}$, and $4^{+}$states of ${ }^{154} \mathrm{Sm}$, respectively. $1 \mathrm{zs}=1 \times 10^{-21} \mathrm{~s}$.

\section{E. Transmission coefficients and the window operator}

We define transmission as any flux that penetrates the Coulomb barrier. After the propagation, the initial and final states $\left|\psi_{i}\right\rangle$ and $\left|\psi_{f}\right\rangle$ are extracted and the transmission coefficients are calculated using

$$
\mathcal{T}(E)=1-\mathcal{R}(E),
$$

where $\mathcal{R}(E)$ is the reflection coefficient given by

$$
\mathcal{R}(E)=\frac{\mathbb{P}_{f}(E)}{\mathbb{P}_{i}(E)}=\frac{\left\langle\psi_{f}|\hat{\Delta}(E)| \psi_{f}\right\rangle}{\left\langle\psi_{i}|\hat{\Delta}(E)| \psi_{i}\right\rangle}
$$

where $\mathbb{P}(E)$ is the probability of a state having energy $E$ and $\hat{\Delta}(E)$ is an energy projection operator. We use an energy projection operator because our propagated wave packets have a broad distribution of energies, but the experimental reactions have sharp energy distributions in the radial motion. In this work, we choose to use the window operator for the energy 

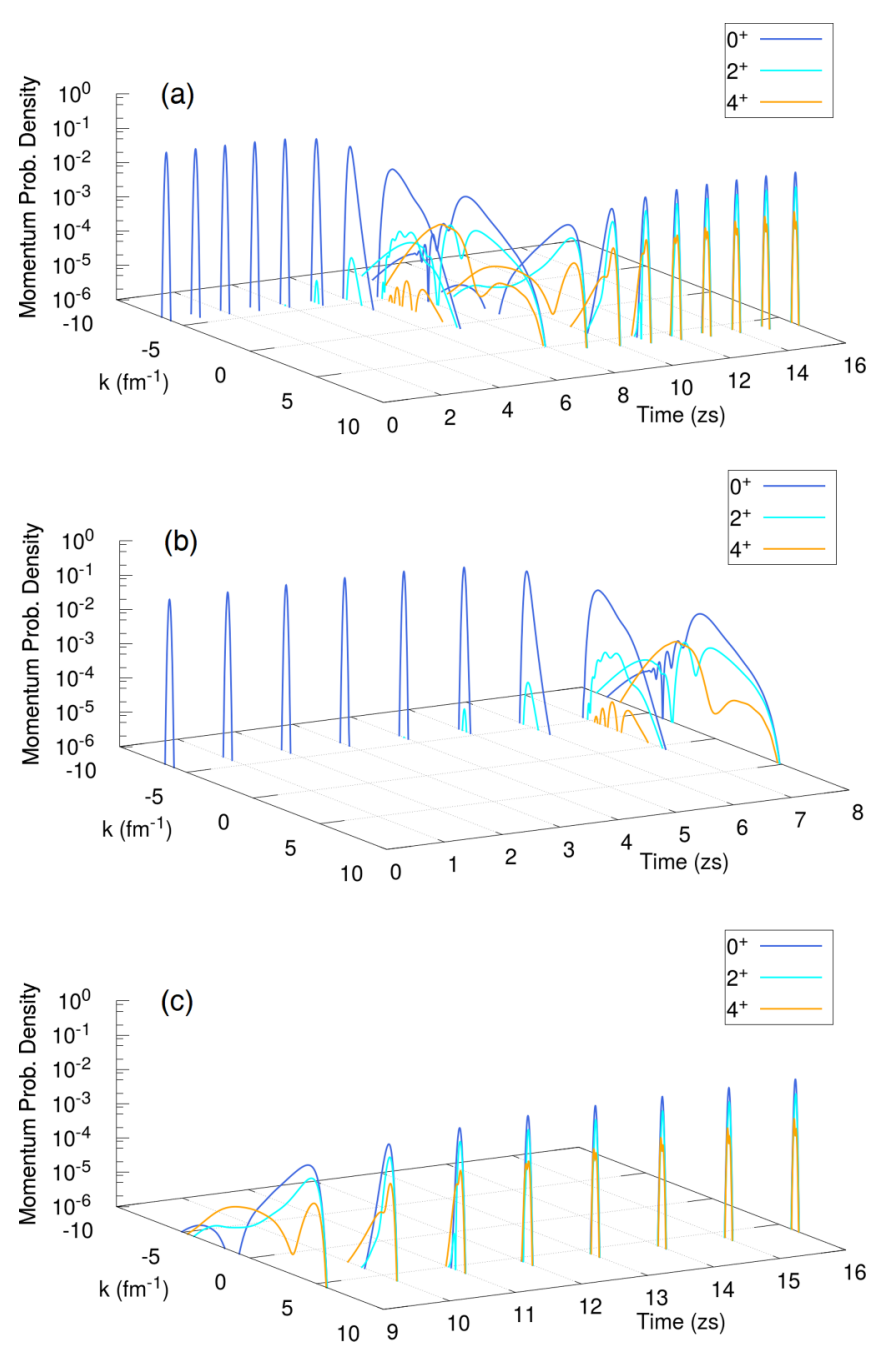

FIG. 3. The same as Fig. 2, but for the momentum probability density.

projection, which is given by $[17,18]$

$$
\hat{\Delta}(E) \equiv \frac{\epsilon^{2^{n}}}{(\hat{H}-E)^{2^{n}}+\epsilon^{2^{n}}},
$$

where $\hat{H}$ is the Hamiltonian of the system, and $n$ is a positive integer that controls the shape of the energy window or bin. With this operator, $\langle\psi|\hat{\Delta}(E)| \psi\rangle$ represents the probability of finding the system, in state $|\psi\rangle$, with energy in the range $E \pm$ $\epsilon$. As $n \rightarrow \infty$, the shape of the bin function rapidly becomes rectangular, with constant bin width of $2 \epsilon$ and little overlap between adjacent energy bins. The spectrum is constructed for a set of energies $E_{k}$ where $E_{k+1}=E_{k}+2 \epsilon$. We can express $\mathbb{P}\left(E_{k}\right)=\left\langle\chi\left(E_{k}\right) \mid \chi\left(E_{k}\right)\right\rangle$, which can be calculated by solving the equation

$$
\left(\left(\hat{H}-E_{k}\right)^{n}-\mathrm{i} \epsilon^{n}\right)\left|\chi\left(E_{k}\right)\right\rangle=\epsilon^{n}|\psi\rangle .
$$

Here, the $\left|\chi\left(E_{k}\right)\right\rangle$ states represent energy-resolved bin states with energy in the range $E_{k} \pm \epsilon$. The accuracy of the window operator is mainly affected by the absolute size of the energy coefficients $\left\langle\phi_{j} \mid \psi\right\rangle$, where $\hat{H}\left|\phi_{j}\right\rangle=\mathcal{E}_{j}\left|\phi_{j}\right\rangle$. That is, if the energy coefficients of state $|\psi\rangle$ in the energy window $E_{k} \pm \epsilon$
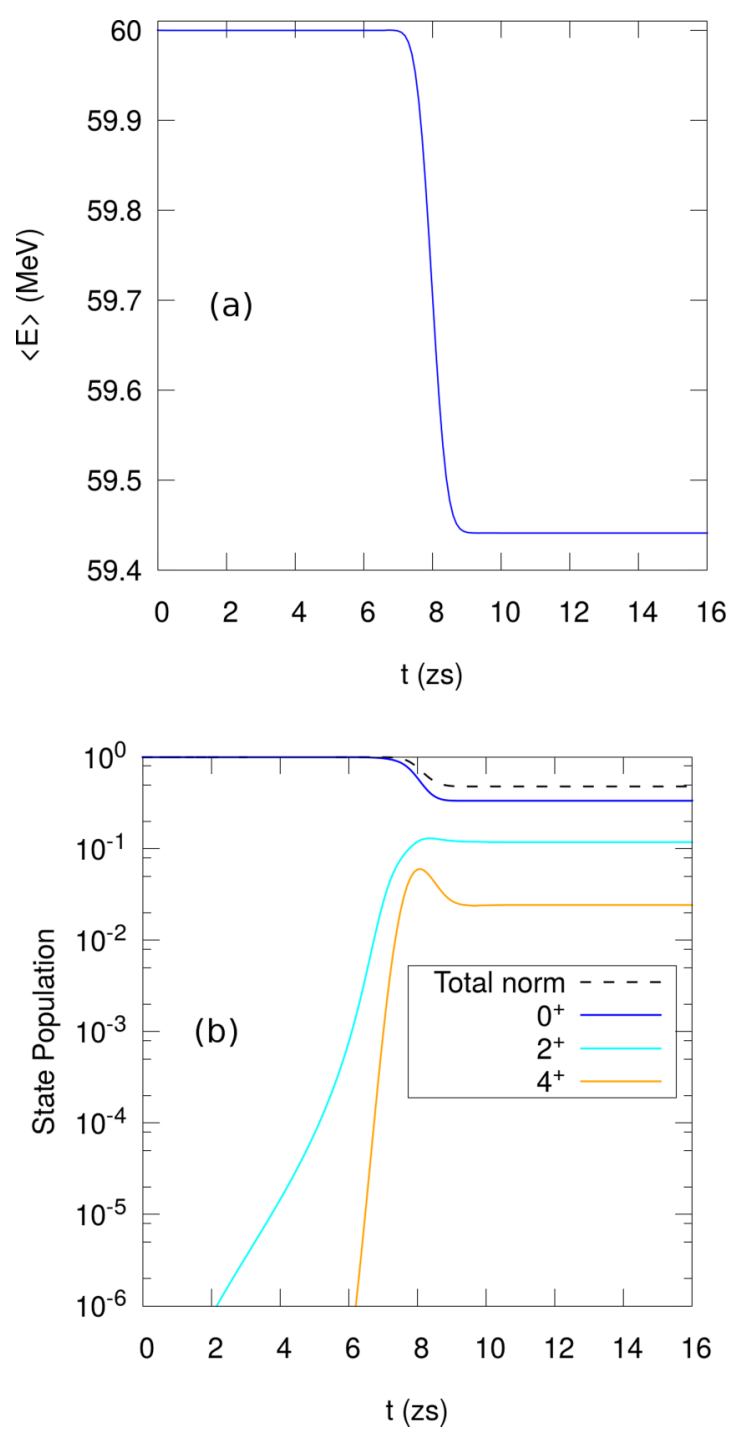

FIG. 4. Expectation energy and wave-function norm over time for $J=0$ and $E_{0}=60 \mathrm{MeV}$. (a) Expectation energy over time. Decrease in the expected energy mirrors absorbed norm in Fig. 4(b). (b) State populations (wave-function norm) over time. The blue (dark grey), cyan (light grey), and orange (grey) lines are the $0^{+}, 2^{+}$, and $4^{+}$state populations of ${ }^{154} \mathrm{Sm}$, respectively, and the black dashed line shows the total state population. Absorption occurs after $6 \mathrm{zs}$, and after $\sim 9$ zs all of the state populations stabilize. The error in the norm before the absorption is of the order of $10^{-14}$.

are too low, then the $\left|\chi\left(E_{k}\right)\right\rangle$ state generated by the window operator calculation will be inaccurate.

\section{RESULTS}

The parameters used in the simulation are given in Tables I and II. The chosen grid parameters are to provide a balanced resolution in the spatial and momentum Fourier grids; a higher resolution spatial grid results in a lower resolution momentum grid and vice versa. The value of $\sigma$ is chosen to ensure that the energy coefficients of the initial wave function $\left|\psi_{0}\right\rangle$ that are 


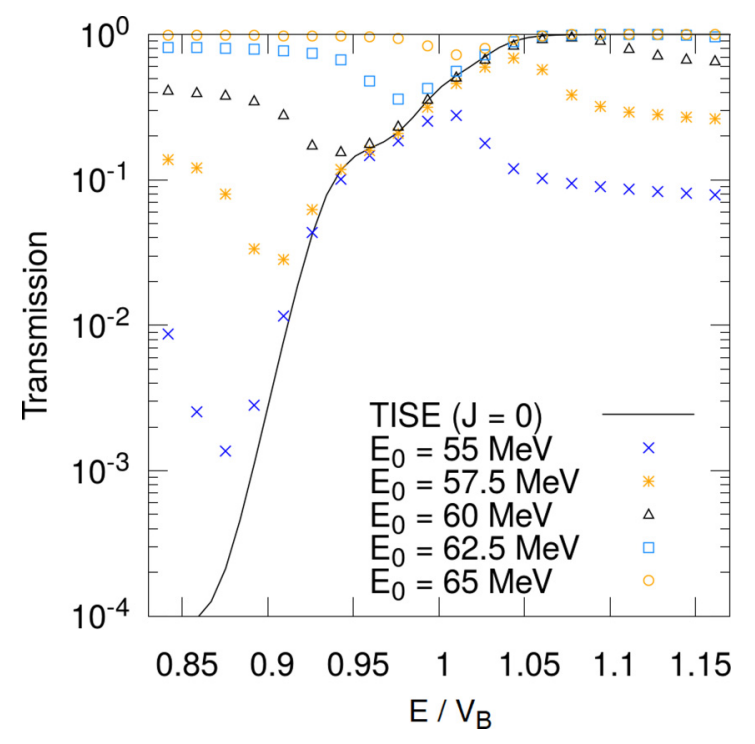

FIG. 5. Energy-resolved transmission coefficients using the window operator method for $J=0$ and the TISE results from using the CCFULL code of Ref. [13]. The profile resulting from the overlap of these $E_{0}$ profiles shows the transmission coefficients (explained in text, shown in Fig. 6). Barrier height for $J=0$ is equal to $59.41 \mathrm{MeV}$.

close to the incident energy $E_{0}$ are high, which allows greater accuracy when using the window operator.

Examples of propagation of the spatial and momentum wave functions for $E_{0}=60 \mathrm{MeV}$ and $J=0$ are shown in Figs. 2 and 3, respectively. We see in Fig. 2(b) that the $2^{+}$and $4^{+}$states penetrate deeper into the Coulomb barrier, because these states are mainly generated from the high momentum states of the $0^{+}$state in the entrance channel. This can be verified in Fig. 3(b), where we see the higher momentum $0^{+}$

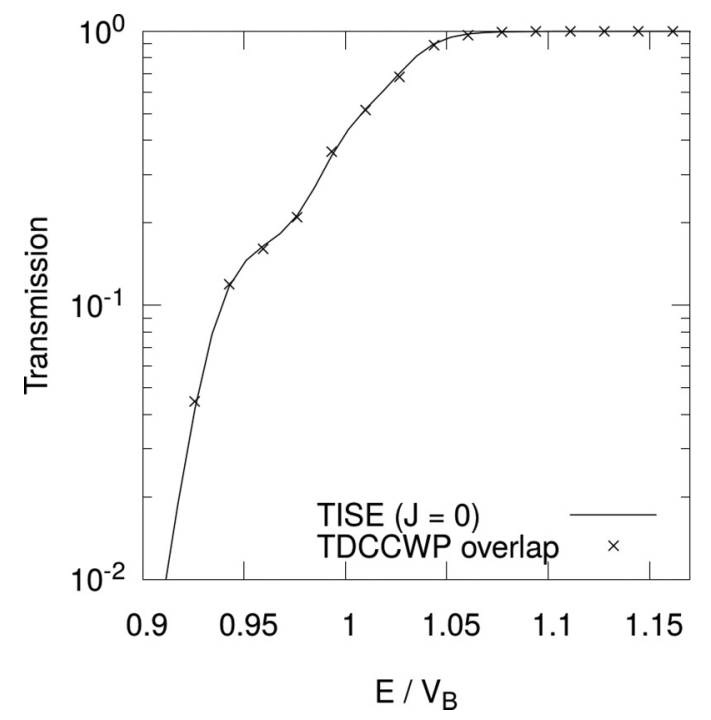

FIG. 6. Overlapped energy-resolved transmission coefficients for $J=0$ and the TISE results from using the CCFULL code of Ref. [13]. Overlapping profile generated by linking $E_{0}$ profiles from Fig. 5 with transmission coefficients that have an absolute difference of $\approx 10^{-2}$.
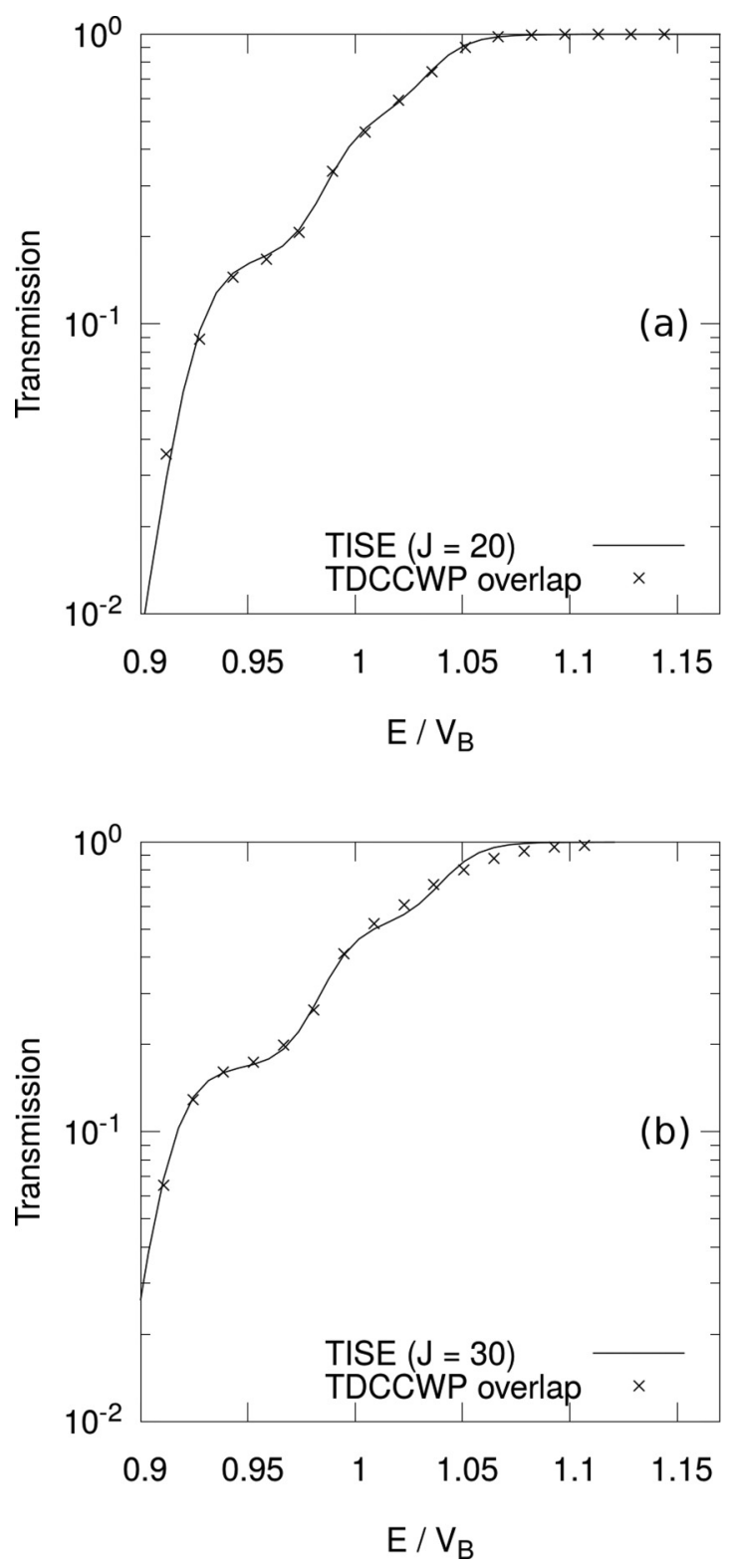

FIG. 7. Overlapped energy-resolved transmission coefficients and the TISE results from using the CCFULL code of Ref. [13] (a) for $J=20$ (barrier height $V_{B}$ equal to $64.69 \mathrm{MeV}$ ) and (b) for $J=30$ (barrier height $V_{B}$ equal to $71.38 \mathrm{MeV}$ ).

states convert into the $2^{+}$and $4^{+}$states at around 6-7 zs. This is physically sensible because the states which penetrate deeper have more momentum and/or energy and are more likely to convert into excited states.

Figures 4(a) and 4(b) show the expectation energy and wave-function norm over time. For a long time before the nuclei are close together, the norm is conserved with an accuracy of $10^{-14}$. The loss of norm due to the absorption is much larger than $10^{-14}$, indicating we can distinguish between the loss of norm due to physical processes and the loss of norm due to computational error. There is a small loss of average energy due to the absorption. 
We confirm that there are no more interactions after at least $10 \mathrm{zs}$ because the momentum distribution of the wave function in Fig. 3(c) is completely located in positive momentum and the wave-function norm in Fig. 4(b) remains constant. Thus for times after at least $10 \mathrm{zs}$ the comparison between the TDWP method and the TISE should yield the same results. The propagation results agree with our expectations.

The energy-resolved transmission coefficients for various incident energies $E_{0}$ are shown in Fig. 5. Physically speaking, the energy-resolved transmission coefficients should not depend on the choice of $E_{0}$ in our model, unlike in Fig. 5. This is due to the limitations of the window operator method when the energy coefficients are low, as described in the last paragraph of Sec. II. The $E_{0}=55-60 \mathrm{MeV}$ curves are inaccurate at higher and lower energies relative to $E_{0}$ since the energy coefficients of $\left|\psi\left(E_{0}\right)\right\rangle$ are low at those energies, and thus the energy-resolved states generated at those energies are inaccurate. Although we are able to describe the transmission accurately for energies several $\mathrm{MeV}$ below the barrier, for energies much further below that the TDWP results differ from the TISE. This low energy behavior is due to the reflection being high, and thus numerical calculations via the reflection coefficient in Eq. (11) are unreliable. The curve produced from the overlap of all of the $E_{0}$ curves in Fig. 5 are the actual transmission coefficients, shown in Fig. 6. This is because for energies where the curves overlap, the transmission values of each curve are calculated using wave packets with high energy coefficients. These overlapping profiles agree well with the expected results from the TISE for a wide range of energies and $J$ values, as shown in Figs. 6 and 7.

\section{SUMMARY}

A new application of the TDCCWP method has been presented. We demonstrate, for the ${ }^{16} \mathrm{O}+{ }^{154} \mathrm{Sm}$ system, that the method calculates transmission coefficients in agreement with the stationary coupled-channels reaction method for a wide range of energies and total angular momenta. Also, we demonstrate that the method provides information about the mechanisms and processes over time, which is less practical to obtain using time-independent methods. The TDWP method is very general and the techniques developed in this work can be applied for the quantitative study of other quantum systems, such as those in different fields, involving barrier tunneling and absorption.

\section{ACKNOWLEDGMENTS}

This work is supported by both the STFC Scholarship Grant (No. 1942568) and the STFC Consolidated Grant (No. ST/P005314/1).
[1] D. J. Tannor, Introduction to Quantum Mechanics: A TimeDependent Perspective (University Science Books, Sausalito, CA, 2007).

[2] R. Kosloff, J. Chem. Phys. 150, 204105 (2019).

[3] B. L. Hu, J. P. Paz, and Y. Zhang, Phys. Rev. D 45, 2843 (1992).

[4] G. Katz, D. Gelman, M. A. Ratner, and R. Kosloff, J. Chem. Phys. 129, 034108 (2008).

[5] J. Dai and J. Z. H. Zhang, J. Phys. Chem. 100, 6898 (1996).

[6] R. D. Levine, Molecular Reaction Dynamics (Cambridge University Press, Cambridge, UK, 2005).

[7] S. Althorpe and D. Clary, Annu. Rev. Phys. Chem. 54, 493 (2003).

[8] B. Zhao and H. Guo, WIREs Comput. Mol. Sci. 7, e1301 (2017).

[9] K. Yabana, M. Ueda, and T. Nakatsukasa, Nucl. Phys. A 722, C261 (2003)
[10] M. Ito, K. Yabana, T. Nakatsukasa, and M. Ueda, Nucl. Phys. A 787, 267 (2007).

[11] A. Diaz-Torres and M. Wiescher, Phys. Rev. C 97, 055802 (2018).

[12] M. Boselli and A. Diaz-Torres, Phys. Rev. C 92, 044610 (2015).

[13] K. Hagino, N. Rowley, and A. Kruppa, Comput. Phys. Commun. 123, 143 (1999).

[14] V. A. Mandelshtam and H. S. Taylor, J. Chem. Phys. 103, 2903 (1995).

[15] A. Diaz-Torres, G. Adamian, V. Sargsyan, and N. Antonenko, Phys. Lett. B 739, 348 (2014).

[16] W. Greiner, J. Maruhn, and D. Bromley, Nuclear Models (Springer, London, 1997).

[17] K. Schafer, Comput. Phys. Commun. 63, 427 (1991).

[18] K. J. Schafer and K. C. Kulander, Phys. Rev. A 42, 5794 (1990). 